\title{
Midpoint-Free Subsets of the Real Numbers
}

\author{
Roger B. Eggleton \\ School of Mathematical and Physical Sciences, University of Newcastle, Callaghan, NSW 2308, Australia
}

Correspondence should be addressed to Roger B. Eggleton; roger@ilstu.edu

Received 22 May 2014; Accepted 29 July 2014; Published 26 August 2014

Academic Editor: Chris A. Rodger

Copyright (C) 2014 Roger B. Eggleton. This is an open access article distributed under the Creative Commons Attribution License, which permits unrestricted use, distribution, and reproduction in any medium, provided the original work is properly cited.

\begin{abstract}
A set of reals $S \subset \mathbb{R}$ is midpoint-free if it has no subset $\{a, b, c\} \subseteq S$ such that $a<b<c$ and $a+c=2 b$. If $S \subset X \subseteq \mathbb{R}$ and $S$ is midpoint-free, it is a maximal midpoint-free subset of $X$ if there is no midpoint-free set $T$ such that $S \subset T \subseteq X$. In each of the cases $X=\mathbb{Z}^{+}, \mathbb{Z}, \mathbb{Q}^{+}, \mathbb{Q}, \mathbb{R}^{+}, \mathbb{R}$, we determine two maximal midpoint-free subsets of $X$ characterised by digit constraints on the base 3 representations of their members.
\end{abstract}

\section{Introduction}

Let us say that a set of three real numbers $\{a, b, c\} \subset \mathbb{R}$ is a midpoint triple if it satisfies $a<b<c$ and $a+c=2 b$. In this case $b$ is the midpoint of the set, $a$ is its lower endpoint, and $c$ is its upper endpoint. This geometric viewpoint immediately suggests the main objective of this paper, which is to identify several significant subsets of $\mathbb{R}$ that contain no midpoint triple.

A midpoint-free subset of $\mathbb{R}$ is any set that contains no midpoint triple. For instance, the set $\left\{2^{n}: n \in \mathbb{Z}\right\}$ of powers of 2 is midpoint-free, since appropriate scaling shows that the sum of two distinct powers of 2 is never equal to a power of 2. Note that if $S \subseteq \mathbb{R}$ and $c S+d:=\{c s+d: s \in S\}$, then $c S+d$ is midpoint-free, for any $\{c, d\} \subset \mathbb{R}$ with $c \neq 0$, if and only if $S$ is midpoint-free.

If $S \subset X \subseteq \mathbb{R}$ and $S$ is midpoint-free, then $S$ is a maximal midpoint-free subset of $X$ if there is a midpoint triple in any set $T$ such that $S \subset T \subseteq X$. (In this case, any $x \in X \backslash S$ is a member of a midpoint triple in $S \cup\{x\}$.) If $X$ is a "natural" subset of $\mathbb{R}$, such as the nonnegative integers $\mathbb{Z}^{+}$, the rationals $\mathbb{Q}$, or indeed $\mathbb{R}$ itself, it is of considerable interest to identify maximal midpoint-free subsets of $X$.

Alternatively, any set $\{a, b, c\}$ such that $a<b<c$ and $a+c=2 b$ can be viewed as a 3-term arithmetic progression (A.P.) with first term $a$, midterm $b$, and last term $c$. This arithmetic viewpoint has led to many studies of sequences of positive integers in which there is no 3-term A.P., raising questions such as the following. How large is a maximal subset of the first $n$ positive integers that contains no 3-term A.P.? (See [1] for data.) Given a finite set $S$ of positive integers with no 3-term A.P., what does its greedy algorithm extension look like? How does it compare with a largest possible subset of the first $n$ positive integers that contains $S$ and has no 3-term A.P.? Under what conditions must a subset of the positive integers contain a 3-term A.P? Among those who have made major contributions to our understanding of these questions, one must list such luminaries as Van der Waerden, Erdős, Turán, Rado, Behrend, Roth, Graham, and Szemerédi. For a compact survey and extensive bibliography of such investigations, see Guy [2]. As an example of recent work in this area, see Dybizbański [3].

In contrast with the usual arithmetic viewpoint, the geometric viewpoint adopted here takes us in an apparently novel direction, where the sets of interest are most naturally viewed as subsets of $\mathbb{R}$.

\section{Notational Conventions}

In what follows, a key tool for discussing midpoint triples will be base 3 representations of the real numbers, so relevant notational conventions will now be specified. If $r \in \mathbb{R}, r>0$ and

$$
r=\sum_{i \in \mathbb{Z}} d_{i} 3^{i} \quad \text { with } d_{i} \in\{0,1,2\}
$$


then the two-way infinite string

$$
\mathbf{d}:=\cdots d_{2} d_{1} d_{0} \cdot d_{-1} d_{-2} \cdots
$$

is a base 3 representation of $r$ and $d_{i}$ is the digit in place $i$ of $\mathbf{d}$. If $d_{i} \in\{0,1\}$ for infinitely many $i<0$, then $\mathbf{d}$ is a regular base 3 representation of $r$; otherwise, $\mathbf{d}$ is a singular base 3 representation of $r$, and there is an integer $k$ such that $d_{i}=2$ for all $i<k$. The regular base 3 representation of $r$ is unique, and if $r$ has a singular base 3 representation, that is also unique. If $X$ is some "natural" subset of $\mathbb{R}$ and $D \subset\{0,1,2\}$, it will be convenient to use $X_{3}(D)$ to denote the set of all members of $X$ with a base 3 representation restricted to $D$. This notation adapts to cases where $D$ is a configuration of base 3 digits; thus, when $D$ is a finite string of base 3 digits, $X_{3}([D])$ will denote the set of all members of $X$ with a base 3 representation which includes a one-way infinite string of recurring blocks $D$.

Since $r>0$, at least one of the digits is nonzero. The leading digit of $\mathbf{d}$ is the digit $d_{h}>0$, such that $d_{i}=0$ for all $i>h$. The trivial zeros of $\mathbf{d}$ are all the digits $d_{i}=0$ with $i>\max \{h, 0\}$. Conventionally these are suppressed (i.e., kept implicit) when listing $\mathbf{d}$. If $h<0$, so the leading digit $d_{h}$ occupies a negative place, the placeholder zeros of $\mathbf{d}$ are all the digits $d_{i}=0$ with $0 \geq i>h$. If there is an integer $k \leq h$ such that $d_{k}>0$ and $d_{i}=0$ for all $i<k$, then $\mathbf{d}$ is a regular representation, $d_{k}$ is its trailing digit, its optional zeros are all the digits $d_{i}=0$ with $i<\min \{k, 0\}$, and if $k>0$ then its placeholder zeros are the digits $d_{i}=0$ with $0 \leq i<k$. If $\mathbf{d}$ has a trailing digit, it is conventional to suppress its optional zeros as well as its trivial zeros; the finite digit string remaining is the terminating base 3 representation of $r$.

These conventions are extended to $r=0$, as follows. Although the digits in this case are $d_{i}=0$ for all $i$, in this exceptional case $d_{0}$ is defined to be both the leading digit and the trailing digit of the representation. Then its trivial zeros are all those in places $i>0$, and its optional zeros are all those in places $i<0$; thus, 0 is the terminating representation for $r=0$.

If $\mathbf{d}$ has no trailing digit, it is a nonterminating representation of $r$ and it may be either regular or singular. If $\mathbf{d}$ is singular, there is an integer $k \leq h+1$ such that $d_{k} \in\{0,1\}$ and $d_{i}=2$ for all $i<k$; the digit $d_{k}$ is the pivot digit of $\mathbf{d}$. In this case there is an alternative, regular base 3 representation of $r$, namely,

$$
\mathbf{e}:=\cdots e_{2} e_{1} e_{0} \cdot e_{-1} e_{-2} \cdots
$$

where $e_{i}=d_{i}$ for all $i>k, e_{k}=d_{k}+1$, and $e_{i}=0$ for all $i<k$. Evidently $\mathbf{e}$ is a terminating representation, $e_{k}$ is its trailing digit, and $3^{-k} r \in \mathbb{Z}^{+}$, so $r \in 3^{k} \mathbb{Z}^{+}$.

The regular base 3 representation of any nonnegative rational $q \in \mathbb{Q}^{+}$has a recurring block of digits; if $q$ has a singular base 3 representation, this has a recurring 2. For brevity, any such recurring block can be enclosed in square brackets, and the subscript 3 can be used to indicate base 3 notation; for instance,

$$
\begin{aligned}
& \frac{7}{3}=2 \cdot 1_{3}=2 \cdot 1[0]_{3}=2 \cdot 0[2]_{3}, \\
& \frac{1}{2}=0 \cdot[1]_{3}, \quad \frac{2}{5}=0 \cdot[1012]_{3} .
\end{aligned}
$$

The set of all nonnegative rationals with a terminating base 3 representation is

$$
\mathbb{Q}_{3}^{+}([0])=\bigcup_{i \in \mathbb{Z}^{+}} 3^{-i} \mathbb{Z}^{+}
$$

The set of positive rationals with a singular base 3 representation is simply

$$
\mathbb{Q}_{3}^{+}([2])=\mathbb{Q}_{3}^{+}([0]) \backslash\{0\} .
$$

The set of positive rationals with base 3 representation containing a recurring 1 is

$$
\mathbb{Q}_{3}^{+}([1])=\bigcup_{i \in \mathbb{Z}^{+}} 3^{-i}\left(\mathbb{Z}^{+}+\frac{1}{2}\right) .
$$

It will later be found that this set requires particular attention when members are doubled, because

$$
2 \mathbb{Q}_{3}^{+}([1]) \subset \mathbb{Q}_{3}^{+}([2]) \subset \mathbb{Q}_{3}^{+}([0]) .
$$

Base 3 representation of negative reals is simply achieved by writing any such number as $-r$, with $r \in \mathbb{R}, r>0$, so that $-r$ has its base 3 representation adopted from that of $r$, with trivial zeros suppressed and the unary minus operator applied to precede the leading digit. If $r$ has both a regular and a singular representation, so does $-r$.

To distinguish between instances of base 3 and base 10 representations, an explicit subscript 3 is normally attached to the former, while a subscript 10 is normally kept implicit for the latter. As needed, $r_{3}$ will denote the regular base 3 representation of $r$, with $r_{3}^{*}$ reserved for the singular representation when this exists. Finally, $\llbracket r \rrbracket_{3, i}$ will denote $d_{i}$, the digit in place $i$ of the regular base 3 representation of $r$, while $\llbracket r \rrbracket_{3, i}^{*}$ will denote the corresponding digit in the singular representation when this exists.

\section{Three Sparse Subsets of $\mathbb{Z}^{+}$}

Let $\mathbb{Z}_{3}^{+}(D)$ be the set of all nonnegative integers with terminating base 3 representation in which the only explicit digits are in $D \subset\{0,1,2\}$. The three cases of interest are those where $D$ is a 2 -set. They begin as follows:

$$
\begin{array}{r}
\mathbb{Z}_{3}^{+}(0,1) \\
=\{0,1,3,4,9,10,12,13,27,28, \\
\quad 30,31,36,37,39,40,81, \ldots\},
\end{array}
$$

$\mathbb{Z}_{3}^{+}(0,2)$

$$
=\{0,2,6,8,18,20,24,26,54,56,60,62,72,74,78,80, \ldots\},
$$




$$
\begin{aligned}
\mathbb{Z}_{3}^{+} & (1,2) \\
& =\{1,2,4,5,7,8,13,14,16,17,22,23,25,26,40, \ldots\} .
\end{aligned}
$$

Some features are obvious. Clearly $\mathbb{Z}_{3}^{+}(0,2)=2 \mathbb{Z}_{3}^{+}(0,1)$. When $d \in\{0,1,2\}$ and $D=\{0,1,2\} \backslash\{d\}$, all integers congruent to $d(\bmod 3)$ are absent from $\mathbb{Z}_{3}^{+}(D)$. The first positive integer absent from all three sets is $102_{3}=11$, but it is clear that an increasing proportion of integers will be missing from all three sets. Indeed, a simple calculation shows for any positive integer $n$ that $\mathbb{Z}_{3}^{+}(0,1)$ and $\mathbb{Z}_{3}^{+}(0,2)$ each have $2^{n}$ members below $3^{n}$, while $\mathbb{Z}_{3}^{+}(1,2)$ has $2^{n+1}-2$ members below $3^{n}$, so each set is sparse and has asymptotic density 0 .

It turns out that these three sets are of considerable interest when it comes to the presence or absence of midpoint triples, so let us now address the main subject of this paper.

Note that the set $\mathbb{Z}_{3}^{+}(1,2)$ contains midpoint triples, such as $\{1,4,7\}$ and $\{2,5,8\}$. In fact, $\mathbb{Z}_{3}^{+}(1,2)$ is densely packed with midpoint triples, since each member $a \in \mathbb{Z}_{3}^{+}(1,2)$ is the lower endpoint of an infinite family of midpoint triples:

$$
\begin{aligned}
& \left\{a, u_{n}-u_{m}+a, 2 u_{n}-2 u_{m}+a\right\} \\
& =\left(u_{n}-u_{m}\right)\{0,1,2\}+a \subset \mathbb{Z}_{3}^{+}(1,2)
\end{aligned}
$$

for any integer $n>m$, where $m$ is the number of digits in the terminating base 3 representation of $a$ (if $d_{h}$ is the leading digit in $a_{3}$, then $m=h+1$ ), and

$$
u_{n}:=1+3+3^{2}+\cdots+3^{n-1}=\frac{3^{n}-1}{2}
$$

is the positive integer with terminating base 3 representation of $n$ digits, all 1 . Note that $\mathbb{Z}_{3}^{+}(1)=\left\{u_{n}: n \geq 1\right\}$, and appropriate scaling shows that this set is midpoint-free. (The integers $u_{n}$ are rep-units in base 3 . The positive integers with explicit decimal digits all equal to 1 are the rep-units for base 10, so called by contraction of "repeated unit" [4]. Prime factorizations of rep-units have been much studied $[5,6]$. Clearly, if $m \mid n$, then $u_{m} \mid u_{n}$. Thus $u_{n}$ can be a prime number only if $n$ is prime, but $u_{5}=11111_{3}=11^{2}$ shows that this condition is not sufficient. Such observations generalize to rep-units in any base.)

Not only is every member of $\mathbb{Z}_{3}^{+}(1,2)$ the lower endpoint of infinitely many midpoint triples, it is also the case that any member greater than 2 is either the midpoint or the upper endpoint of at least one midpoint triple, depending on the leading digit of its base 3 representation.

In contrast, it turns out that $\mathbb{Z}_{3}^{+}(0,1)$ and $\mathbb{Z}_{3}^{+}(0,2)$ are midpoint-free. To see this, it suffices to show that $\mathbb{Z}_{3}^{+}(0,1)$ is midpoint-free: then $\mathbb{Z}_{3}^{+}(0,2)=2 \mathbb{Z}_{3}^{+}(0,1)$ ensures that $\mathbb{Z}_{3}^{+}(0,2)$ is also midpoint-free. (Long ago, Erdős and Turán [7] noted that the nonnegative integers with a 2-less base 3 representation are midpoint-free. The following compact proof is included for completeness and to typify what follows.)

Claim $A$. The set $\mathbb{Z}_{3}^{+}(0,1)$ is midpoint-free.
Proof. On the contrary, suppose that $\{a, b, c\} \subset \mathbb{Z}_{3}^{+}(0,1)$ is a midpoint triple, with $a<b<c$ and $a+c=2 b$. All digits in $a_{3}, b_{3}, c_{3}$ are in $\{0,1\}$ and all digits in $(2 b)_{3}$ are in $\{0,2\}$. Then

$$
\llbracket a \rrbracket_{3, i}+\llbracket c \rrbracket_{3, i}=\llbracket 2 b \rrbracket_{3, i}
$$

for all integers $i$, and

$$
\begin{aligned}
& \llbracket 2 b \rrbracket_{3, i}=0 \Longrightarrow \llbracket a \rrbracket_{3, i}=\llbracket b \rrbracket_{3, i}=\llbracket c \rrbracket_{3, i}=0, \\
& \llbracket 2 b \rrbracket_{3, i}=2 \Longrightarrow \llbracket a \rrbracket_{3, i}=\llbracket b \rrbracket_{3, i}=\llbracket c \rrbracket_{3, i}=1 .
\end{aligned}
$$

Hence, $a=b=c$, a contradiction, so $\mathbb{Z}_{3}^{+}(0,1)$ contains no midpoint triple.

It will now be shown that $\mathbb{Z}_{3}^{+}(0,1)$ is not contained in any larger midpoint-free subset of $\mathbb{Z}^{+}$.

Claim $B$. For any positive integer $b \in \mathbb{Z}^{+} \backslash \mathbb{Z}_{3}^{+}(0,1)$, there is a midpoint triple in $\mathbb{Z}_{3}^{+}(0,1) \cup\{b\}$ with $b$ as its midpoint.

Proof. Given $b \in \mathbb{Z}^{+} \backslash \mathbb{Z}_{3}^{+}(0,1)$, we seek $\{a, c\} \subset \mathbb{Z}_{3}^{+}(0,1)$ such that $a<b<c$ and $a+c=2 b$. Specify the base 3 digits of $a$ and $c$ as follows:

$$
\begin{gathered}
\llbracket 2 b \rrbracket_{3, i}=0 \Longrightarrow \llbracket a \rrbracket_{3, i}=\llbracket c \rrbracket_{3, i}=0, \\
\llbracket 2 b \rrbracket_{3, i}=2 \Longrightarrow \llbracket a \rrbracket_{3, i}=\llbracket c \rrbracket_{3, i}=1, \\
\llbracket 2 b \rrbracket_{3, i}=1 \Longrightarrow \llbracket a \rrbracket_{3, i}=0, \quad \llbracket c \rrbracket_{3, i}=1 .
\end{gathered}
$$

This determines integers $a$ and $c$ such that $\{a, c\} \subset \mathbb{Z}_{3}^{+}(0,1)$ and $a+c=2 b$ since

$$
\llbracket a \rrbracket_{3, i}+\llbracket c \rrbracket_{3, i}=\llbracket 2 b \rrbracket_{3, i},
$$

for all $i$. At least one digit of $(2 b)_{3}$ is 1 , since $b \notin \mathbb{Z}_{3}^{+}(0,1)$, so $a_{3}$ and $c_{3}$ differ in at least one place: then $\llbracket a \rrbracket_{3, i}<\llbracket c \rrbracket_{3, i}$ in each such place, ensuring that $a<c$. Put $d:=(c-a) / 2>0$. Then $c=a+2 d$, so $2 b=a+c=2 a+2 d$, whence $b=a+d>a$ and $c=a+2 d=b+d>b$. Thus $a<b<c$.

It will now be shown that extending $\mathbb{Z}_{3}^{+}(0,1)$ by adjoining any rational of the form $n+(1 / 2)$, with $n \in \mathbb{Z}^{+}$, always yields a set which contains a midpoint triple.

Claim $C$. For any $b \in \mathbb{Z}^{+}+(1 / 2)$, there is a midpoint triple in $\mathbb{Z}_{3}^{+}(0,1) \cup\{b\}$ with $b$ as its midpoint.

Proof. Again we seek $\{a, c\} \subset \mathbb{Z}_{3}^{+}(0,1)$, such that $a<b<c$ and $a+c=2 b$. In the present case, note that $2 b$ is an odd positive integer, so $(2 b)_{3}$ contains the digit 1 an odd number of times. Specifying $a$ and $c$ as in the proof of Claim B, once again it follows that $\{a, c\} \subset \mathbb{Z}_{3}^{+}(0,1)$ and $a<b<c$.

For instance, the proofs of Claims B and C produce the decompositions

$$
1201_{3}=100_{3}+1101_{3}, \quad 212_{3}=101_{3}+111_{3}
$$

showing that 23 and $23 / 2$ are the midpoints of the pairs $\{9,37\} \subset \mathbb{Z}_{3}^{+}(0,1)$ and $\{10,13\} \subset \mathbb{Z}_{3}^{+}(0,1)$, respectively. 
Further, doubling shows that 46 and 23 are the midpoints of the pairs $\{18,74\} \subset \mathbb{Z}_{3}^{+}(0,2)$ and $\{20,26\} \subset \mathbb{Z}_{3}^{+}(0,2)$, respectively. From Claims $\mathrm{A}, \mathrm{B}$, and $\mathrm{C}$ and the doubling method just illustrated, we have the following.

Theorem 1. The sets $\mathbb{Z}_{3}^{+}(0,1)$ and $\mathbb{Z}_{3}^{+}(0,2)$ are maximal midpoint-free subsets of $\mathbb{Z}^{+}$.

\section{Two Unexpected Results for $\mathbb{Z}$}

Let us now study $\mathbb{Z}_{3}^{+}(0,1)$ and $\mathbb{Z}_{3}^{+}(0,2)$ as midpoint-free subsets of $\mathbb{Z}$, the full set of integers. Surprisingly, the results for the two sets are quite different.

Calculations until now have involved sums of pairs $\{a, c\} \subset \mathbb{Z}_{3}^{+}(0,1)$, so it has been possible to work digit by digit in base 3 without a carry over digit. When the context is widened to $\mathbb{Z}$, it seems that carry over digits can no longer be avoided, so we shall consider blocks of digits rather than single digits. Let the compact notation $d^{\times n}$ (" $d$ by $n$ ") denote a homogeneous block of $n$ adjacent digits all equal to $d$; thus, $1^{\times n}$ denotes the terminating base 3 representation of rep-unit $u_{n}$. For blocks in which the digits are not all equal we simply abut such expressions with $d^{\times 1}=d$ as a natural simplification. To illustrate, consider the integer

$$
a_{3}:=1222202111001121100=12^{\times 4} 021^{\times 3} 0^{\times 2} 1^{\times 2} 21^{\times 2} 0^{\times 2} .
$$

Let us find integers $b, c \in \mathbb{Z}_{3}^{+}(0,1)$ such that $a+2 b=c$. Regarding $a_{3}$ as a string of homogeneous blocks, we choose matching blocks for $(2 b)_{3}$ so that

$$
(2 b)_{3}:=2000020222222202200=20^{\times 4} 202^{\times 7} 02^{\times 2} 0^{\times 2} .
$$

Calculating from right to left, base 3 arithmetic for $a_{3}+(2 b)_{3}$ yields successively the following blocks, with each carry over digit indicated parenthetically:

$$
\begin{gathered}
0^{\times 2}+0^{\times 2}=0^{\times 2}, \\
1^{\times 2}+2^{\times 2}=(1) 10, \\
2+0+(1)=(1) 0, \\
1^{\times 2}+2^{\times 2}+(1)=(1) 1^{\times 2}, \\
0^{\times 2}+2^{\times 2}+(1)=(1) 0^{\times 2}, \\
1^{\times 3}+2^{\times 3}+(1)=(1) 1^{\times 3}, \\
2+0+(1)=(1) 0, \\
0+2+(1)=(1) 0, \\
2^{\times 4}+0^{\times 4}+(1)=(1) 0^{\times 4}, \\
1+2+(1)=(1) 1 .
\end{gathered}
$$

Assembling these blocks yields

$$
a_{3}+(2 b)_{3}=1^{\times 2}\left|0^{\times 4}\right| 0|0| 1^{\times 3}\left|0^{\times 2}\right| 1^{\times 2}|0| 10 \mid 0^{\times 2} \text {. }
$$

After simplifying we have

$$
\begin{gathered}
10^{\times 4} 101^{\times 7} 01^{\times 2} 0^{\times 2}=1000010111111101100=b_{3}, \\
1^{\times 2} 0^{\times 6} 1^{\times 3} 0^{\times 2} 1^{\times 2} 010^{\times 3}=11000000111001101000=c_{3},
\end{gathered}
$$

so $a+2 b=c$ and $b, c \in \mathbb{Z}_{3}^{+}(0,1)$ as desired. This calculation models the proof of the following.

Claim D. For any integer $a>0$, there is a midpoint triple in $\mathbb{Z}_{3}^{+}(0,1) \cup\{-a\}$ with the negative integer $-a$ as its lower endpoint.

Proof. Given any positive integer $a$, we seek $\{b, c\} \subset \mathbb{Z}_{3}^{+}(0,1)$, such that $-a<b<c$ and $-a+c=2 b$, so $a+2 b=c$. Partition $a_{3}$ into its homogeneous blocks $A_{i}$, so

$$
a_{3}=A_{m} \cdots A_{i} \cdots A_{1} A_{0}
$$

and seek corresponding blocks $B_{i}$ and $C_{i}$ to construct $b_{3}$ and $c_{3}$.

If $a_{3}$ has a terminal block $A_{0}=0^{\times n}$, assign $b_{3}$ the terminal block $B_{0}=0^{\times n}$, so $c_{3}$ has the terminal block $C_{0}=0^{\times n}=$ $A_{0}+2 B_{0}$. If $a_{3}$ has a nonterminal block $A_{i}=0^{\times n}$ for some $i>0$, assume the sum of the preceding blocks $A_{i-1}$ and $2 B_{i-1}$ contributes a carry over digit of 1 , and assign $b_{3}$ the corresponding block $B_{i}=1^{\times n}$, so

$$
C_{i}=A_{i}+2 B_{i}+(1)=0^{\times n}+2^{\times n}+(1)=(1) 0^{\times n} .
$$

As $2^{\times n}+(1)$ corresponds to $2 u_{n}+1=\left(3^{n+1}-1\right)+1=3^{n+1}$, the computation is justified.

If $a_{3}$ has a block $A_{i}=1^{\times n}$, assign $b_{3}$ the corresponding block $B_{i}=1^{\times n}$, so $c_{3}$ has the corresponding block $C_{i}=A_{i}+$ $2 B_{i}+(\delta)=(1) 1^{\times(n-1)} 0+(\delta)$, where the carry over digit $\delta$ is either 0 or 1 , so $C_{i}=(1) 1^{\times(n-1)} 0$ or (1) $1^{\times n}$, respectively. This holds since the sum $1^{\times n}+2^{\times n}$ corresponds to $u_{n}+2 u_{n}=3 u_{n}=$ $u_{n+1}-u_{1}$ and $1^{\times n}+2^{\times n}+(1)$ corresponds to $3 u_{n}+1=u_{n+1}$.

If $a_{3}$ has a terminal block $A_{0}=2^{\times n}$, assign $b_{3}$ the block $B_{0}=0^{\times(n-1)} 1$, so $c_{3}$ has terminal block $C_{0}=A_{0}+2 B_{0}=$ (1) $0^{\times(n-1)} 1$, since the sum $2^{\times n}+0^{\times(n-1)} 2$ corresponds to $2 u_{n}+$ $2=\left(3^{n+1}-1\right)+2=3^{n+1}+1$. Similarly if $A_{1} A_{0}=2^{\times n} 0^{\times m}$, assign $b_{3}$ the corresponding blocks $B_{1} B_{0}=0^{\times(n-1)} 10^{\times m}$; then $c_{3}$ has the blocks $C_{1} C_{0}=(1) 0^{\times(n-1)} 10^{\times m}$. Otherwise, if $a_{3}$ has a nonterminal block $A_{i}=2^{\times n}$ for some $i>0$, assume that $A_{i-1}+2 B_{i-1}$ yields a carry over digit 1 , and assign $b_{3}$ the block $B_{i}=0^{\times n}$, so $c_{3}$ has $C_{i}=A_{i}+2 B_{i}+(1)=(1) 0^{\times n}$.

For $i>0$ note that the only case in which $A_{i-1}+2 B_{i-1}$ does not contribute a carry over digit 1 to $A_{i}+2 B_{i}$ is when $i=1$ and $a_{3}$ has terminal block $A_{0}=0^{\times n}$. Thus, all our assumptions about carry over digits are justified $a$ posteriori.

Claim E. For any integer $n>0$, there is a midpoint triple in $\mathbb{Z}_{3}^{+}(0,2) \cup\{-n\}$ if and only if $n$ is even, and then $-n$ is the lower endpoint of the triple.

Proof. By doubling, it follows from Claim D that, for any integer $a>0$, there is a midpoint triple in $\mathbb{Z}_{3}^{+}(0,2) \cup$ 
$\{-2 a\}$. However, we claim that there is no midpoint triple in $\mathbb{Z}_{3}^{+}(0,2) \cup\{-2 a+1\}$. On the contrary, suppose $-2 a+1$ forms a midpoint triple with the pair $\{b, c\} \subset \mathbb{Z}_{3}^{+}(0,2)$. Without loss of generality, $-2 a+1<b<c$ and $(-2 a+1)+c=2 b$, so $2 a+2 b-1=c$ : this is impossible, since $2 a+2 b-1$ is odd and $c$ is even. It follows that $\mathbb{Z}_{3}^{+}(0,2) \cup\{-2 a+1\}$ is midpointfree.

Claim $F$. The set $\mathbb{Z}_{3}^{+}(0,2) \cup-\left(\mathbb{Z}_{3}^{+}(0,2)+1\right)$ is midpoint-free.

Proof. The two sets forming this union are certainly midpoint-free, so any midpoint triple in the union must have two members in one set and one member in the other. Claim E shows that there is no midpoint triple with exactly one member in $-\left(\mathbb{Z}_{3}^{+}(0,2)+1\right)$, since this set only contains odd negative integers. So suppose there is a midpoint triple $\{-a,-b, c\}$ with exactly one member $c \in \mathbb{Z}_{3}^{+}(0,2)$. Then $\{a-1, b-1,-c-1\}$ is a midpoint triple in

$$
\begin{gathered}
-\left(\mathbb{Z}_{3}^{+}(0,2) \cup-\left(\mathbb{Z}_{3}^{+}(0,2)+1\right)\right)-1 \\
=\mathbb{Z}_{3}^{+}(0,2) \cup-\left(\mathbb{Z}_{3}^{+}(0,2)+1\right)
\end{gathered}
$$

with exactly one member in $-\left(\mathbb{Z}_{3}^{+}(0,2)+1\right)$, the case ruled out by Claim E.

Since $\mathbb{Z}_{3}^{+}(0,2)$ is a maximal midpoint-free subset of $\mathbb{Z}^{+}$ by Theorem 1 , clearly $\mathbb{Z}_{3}^{+}(0,2)+1$ is a maximal midpointfree subset of $\mathbb{Z}^{+}+1=\mathbb{Z}^{+} \backslash\{0\}$. Note that the members of $\mathbb{Z}_{3}^{+}(0,2)+1$ are precisely those positive integers $n$ for which the trailing digit of $n_{3}$ is 1 , and all other digits are in $\{0,2\}$. With Claims $\mathrm{E}$ and $\mathrm{F}$, it follows that $\mathbb{Z}_{3}^{+}(0,2) \cup-\left(\mathbb{Z}_{3}^{+}(0,2)+1\right)$ is a maximal midpoint-free subset of $\mathbb{Z}=\mathbb{Z}^{+} \cup-\left(\mathbb{Z}^{+} \backslash\{0\}\right)$. Combined with Claim D, this proves the following.

Theorem 2. The sets $\mathbb{Z}_{3}^{+}(0,1)$ and $\mathbb{Z}_{3}^{+}(0,2) \cup-\left(\mathbb{Z}_{3}^{+}(0,2)+1\right)$ are maximal midpoint-free subsets of $\mathbb{Z}$.

\section{Midpoint-Free Subsets of $\mathbb{Q}^{+}$}

Now let us consider the corresponding subsets $\mathbb{Q}_{3}^{+}(D)$ of the nonnegative rationals $\mathbb{Q}^{+}$, namely, the subsets comprising those members with a regular base 3 representation in which each digit, after suppression of all trivial and optional zeros, is in $D \subset\{0,1,2\}$. The three cases of interest are those where $D$ is a 2 -set.

As expected, $\mathbb{Q}_{3}^{+}(1,2)$ is densely packed with midpoint triples. This follows from the observation that each rational $q \in \mathbb{Q}_{3}^{+}(1,2)$ is the lower endpoint of an infinite family of midpoint triples,

$$
\begin{aligned}
\{q & \left., u_{n}-u_{m}+q, 2 u_{n}-2 u_{m}+q\right\} \\
\quad= & \left(u_{n}-u_{m}\right)\{0,1,2\}+q \subset \mathbb{Q}_{3}^{+}(1,2)
\end{aligned}
$$

for any integer $n>m$, where $u_{n}$ is the $n$-digit base 3 rep-unit and $m=h+1$, where $d_{h}$ is the leading digit of the regular base 3 representation $q_{3}$.

Next, consider $\mathbb{Q}_{3}^{+}(0,1)$. The details are a little more complicated than in the integer context, since allowance must be made for singular representations when particular members of $\mathbb{Q}_{3}^{+}(0,1)$ are doubled.

Claim $G$. The set $\mathbb{Q}_{3}^{+}(0,1) \backslash \mathbb{Q}_{3}^{+}([1])$ is midpoint-free.

Proof. If $b \in \mathbb{Q}_{3}^{+}(0,1) \backslash \mathbb{Q}_{3}^{+}([1])$ and $b>0$, all digits of $(2 b)_{3}$ are in $\{0,2\}$. They uniquely determine the digits of $a_{3}$ and $c_{3}$ such that $\{a, c\} \subset \mathbb{Q}_{3}^{+}(0,1) \backslash \mathbb{Q}_{3}^{+}([1])$ and $a+c=2 b$. As in the proof of Claim A, they force $a=b=c$. Hence, there is no midpoint triple $\{a, b, c\} \subset \mathbb{Q}_{3}^{+}(0,1) \backslash \mathbb{Q}_{3}^{+}([1])$.

Claim $H$. Any $b \in \mathbb{Q}_{3}^{+}([1])$ is the midpoint of a triple with both its endpoints in the set $\mathbb{Q}_{3}^{+}(0,1) \cap \mathbb{Q}_{3}^{+}([0])$.

Proof. We seek $\{a, c\} \subset \mathbb{Q}_{3}^{+}(0,1) \backslash \mathbb{Q}_{3}^{+}([1])$ with $a<b<c$ and $a+c=2 b$. Then $a_{3}$ and $c_{3}$ are uniquely determined by

$$
\llbracket a \rrbracket_{3, i}+\llbracket c \rrbracket_{3, i}=\llbracket 2 b \rrbracket_{3, i}, \quad \llbracket a \rrbracket_{3, i} \leq \llbracket c \rrbracket_{3, i}
$$

for all $i$. Since $b \in \mathbb{Q}_{3}^{+}([1])$, then $3^{k} b \in \mathbb{Z}^{+}+(1 / 2)$ for some $k \in \mathbb{Z}^{+}$, so

$$
2 b \in 3^{-k}\left(2 \mathbb{Z}^{+}+1\right) .
$$

It follows that $(2 b)_{3}$ is a terminating representation with an odd number of digits equal to 1 . Then

$$
\begin{gathered}
\llbracket 2 b \rrbracket_{3, i}=1 \Longrightarrow \llbracket a \rrbracket_{3, i}=0, \quad \llbracket c \rrbracket_{3, i}=1 \\
\text { for an odd number of integers } i \geq-k ; \\
\llbracket 2 b \rrbracket_{3, i}=0 \Longrightarrow \llbracket a \rrbracket_{3, i}=\llbracket c \rrbracket_{3, i}=0 \quad \forall i<-k .
\end{gathered}
$$

Hence, the required midpoint triple $\{a, b, c\}$ exists, and in fact $\{a, c\} \subset \mathbb{Q}_{3}^{+}([0])$.

In particular, if $b \in \mathbb{Q}_{3}^{+}([1])$ is such that $\llbracket 2 b \rrbracket_{3, n}=1$ for some integer $n$ and $\llbracket 2 b \rrbracket_{3, i}=0$ for all $i \neq n$, then $b=3^{n} / 2=$ $u_{n}+(1 / 2)$ and the proof of Claim H determines $a=0$ and $c=2 b=3^{n}$. Since $\llbracket b \rrbracket_{3, i}=0$ if $i \geq n$ and $\llbracket b \rrbracket_{3, i}=1$ if $i<n$, we call $b$ the base 3 fractional rep-unit with offset $n$, denoted by $v_{n}$. Then $v_{n} \in \mathbb{Q}_{3}^{+}([1])$ is the midpoint of the triple with endpoints $\left\{0,3^{n}\right\} \subset \mathbb{Q}_{3}^{+}(0,1) \cap \mathbb{Q}_{3}^{+}([0])$.

Positive rationals not in $\mathbb{Q}_{3}^{+}(0,1) \cup \mathbb{Q}_{3}^{+}([1])$ also belong to midpoint triples with endpoints in $\mathbb{Q}_{3}^{+}(0,1) \backslash \mathbb{Q}_{3}^{+}([1])$, but proving this needs care. For instance,

$$
\begin{aligned}
\frac{5}{16} & =0 \cdot[0221]_{3} \\
& \Longrightarrow \frac{5}{8}=0 \cdot[12]_{3}=0 \cdot[0111]_{3}+0 \cdot[1101]_{3} \\
& =\frac{13}{80}+\frac{37}{80},
\end{aligned}
$$

so $\{13 / 80,5 / 16,37 / 80\}$ is a midpoint triple with endpoints in $\mathbb{Q}_{3}^{+}(0,1) \backslash \mathbb{Q}_{3}^{+}([1])$. This calculation models the proof of the following general result.

Claim I. Any $b \in \mathbb{Q}^{+} \backslash \mathbb{Q}_{3}^{+}(0,1)$ is the midpoint of a triple with both its endpoints in the set $\mathbb{Q}_{3}^{+}(0,1) \backslash \mathbb{Q}_{3}^{+}([1])$. 
Proof. We seek $\{a, c\} \subset \mathbb{Q}_{3}^{+}(0,1) \backslash \mathbb{Q}_{3}^{+}([1])$ with $a<b<c$ and $a+c=2 b$. When $b \in \mathbb{Q}_{3}^{+}([1])$, the result follows from Claim $\mathrm{H}$, so we may now assume that

$$
b \in \mathbb{Q}^{+} \backslash\left(\mathbb{Q}_{3}^{+}(0,1) \cup \mathbb{Q}_{3}^{+}([1])\right) .
$$

Then $(2 b)_{3}$ has at least one digit $d_{j}$ equal to 1 and its recurring block, say $[D]$, has at least one digit different from 2 . We require $a_{3}$ and $c_{3}$ to satisfy

$$
\llbracket a \rrbracket_{3, i}+\llbracket c \rrbracket_{3, i}=\llbracket 2 b \rrbracket_{3, i}
$$

for all $i$. Requiring $\llbracket a \rrbracket_{3, i} \leq \llbracket c \rrbracket_{3, i}$ for all $i \geq j$ ensures that $a<c$, since

$$
\llbracket a \rrbracket_{3, j}=0, \quad \llbracket c \rrbracket_{3, j}=1 .
$$

If the recurring block $[D]$ of $(2 b)_{3}$ contains 0 , then the recurring blocks of $a_{3}$ and $c_{3}$ also contain 0 , so neither $a$ nor $c$ is in $\mathbb{Q}_{3}^{+}([1])$. Now suppose $[D]$ has at least one digit equal to 1 and none equal to 0 . Clearly, in this case we may choose the integer $j$ so that the digit $d_{j}=1$ occurs in $[D]$. If $D$ has $m$ digits, then for all $i \leq j$ choose

$$
\begin{gathered}
\llbracket a \rrbracket_{3, i}=0, \quad \llbracket c \rrbracket_{3, i}=1 \quad \text { when } i \equiv j \bmod 2 m, \\
\llbracket a \rrbracket_{3, i}=1, \quad \llbracket c \rrbracket_{3, i}=0 \quad \text { when } i \equiv j+m \bmod 2 m \\
\llbracket a \rrbracket_{3, i} \leq \llbracket c \rrbracket_{3, i} \quad \text { otherwise. }
\end{gathered}
$$

Then $a_{3}$ and $c_{3}$ are uniquely determined, so that $a<c$ and each has a recurring block containing 0 , so neither $a$ nor $c$ is in $\mathbb{Q}_{3}^{+}([1])$.

If $q \in \mathbb{Q}^{+} \backslash \mathbb{Q}_{3}^{+}(0,2)$ then $q_{3}$ has at least one digit equal to 1 , so it follows that $(q / 2)_{3} \in \mathbb{Q}^{+} \backslash \mathbb{Q}_{3}^{+}(0,1)$. Claim I shows that there is a midpoint triple $\{a, b, c\}$ with $b=q / 2$ and $\{a, c\} \subset \mathbb{Q}_{3}^{+}(0,1) \backslash \mathbb{Q}_{3}^{+}([1])$. Then $2\{a, b, c\}$ is a midpoint triple with midpoint $q$ and endpoints $2\{a, c\} \subset \mathbb{Q}_{3}^{+}(0,2)$. Hence, doubling applied to Claims $G$ and I shows that $\mathbb{Q}_{3}^{+}(0,2)$ is midpoint-free, and every $b \in \mathbb{Q}^{+} \backslash \mathbb{Q}_{3}^{+}(0,2)$ is the midpoint of a triple with endpoints in $\mathbb{Q}_{3}^{+}(0,2)$, so Claims $G, H$, and I establish the following.

Theorem 3. The sets $\mathbb{Q}_{3}^{+}(0,1) \backslash \mathbb{Q}_{3}^{+}([1])$ and $\mathbb{Q}_{3}^{+}(0,2)$ are maximal midpoint-free subsets of $\mathbb{Q}^{+}$.

\section{Midpoint-Free Subsets of $\mathbb{Q}$}

Now consider $\mathbb{Q}_{3}^{+}(0,1)$ and $\mathbb{Q}_{3}^{+}(0,2)$ as subsets of $\mathbb{Q}$. The role of $\mathbb{Q}_{3}^{+}([1])$ is yet more prominent in this context.

Claim J. The set $\mathbb{Q}_{3}^{+}(0,1) \cap \mathbb{Q}_{3}^{+}([1])$ is midpoint-free.

Proof. Suppose $\{a, b, c\} \subset \mathbb{Q}_{3}^{+}(0,1) \cap \mathbb{Q}_{3}^{+}([1])$ satisfies $a<$ $b<c$ and $a+c=2 b$. There is an integer $m \geq 0$ for which $3^{m}\{a, b, c\} \subset \mathbb{Z}_{3}^{+}(0,1)+(1 / 2)$ so

$$
2 \cdot 3^{m}\{a, b, c\} \subset 2 \mathbb{Z}_{3}^{+}(0,1)+1 .
$$

This contradicts the fact that $2 \mathbb{Z}_{3}^{+}(0,1)+1$ is midpoint-free, by Claim A.
Claim K. Any positive rational $b \in \mathbb{Q}_{3}^{+}([1]) \backslash \mathbb{Q}_{3}^{+}(0,1)$ is the midpoint of a triple with endpoints in $\mathbb{Q}_{3}^{+}(0,1) \cap \mathbb{Q}_{3}^{+}([1])$.

Proof. Given $b$, we seek $\{a, c\} \subset \mathbb{Q}_{3}^{+}(0,1) \cap \mathbb{Q}_{3}^{+}([1])$, such that $a<b<c$ and $a+c=2 b$. There is an integer $m$ such that $3^{m} b \in \mathbb{Z}^{+}+(1 / 2)$, so there is an integer $B \in \mathbb{Z}^{+} \backslash \mathbb{Z}_{3}^{+}(0,1)$ such that $3^{m} b=B+(1 / 2)$. At least one digit of $B_{3}$ is 2 , so at least one digit of $(2 B)_{3}$ is 1 . Define $\{A, C\} \subset \mathbb{Z}_{3}^{+}(0,1)$ by

$$
\begin{gathered}
\llbracket 2 B \rrbracket_{3, i}=0 \Longrightarrow \llbracket A \rrbracket_{3, i}=\llbracket C \rrbracket_{3, i}=0, \\
\llbracket 2 B \rrbracket_{3, i}=1 \Longrightarrow \llbracket A \rrbracket_{3, i}=0, \quad \llbracket C \rrbracket_{3, i}=1, \\
\llbracket 2 B \rrbracket_{3, i}=2 \Longrightarrow \llbracket A \rrbracket_{3, i}=\llbracket C \rrbracket_{3, i}=1 .
\end{gathered}
$$

Then $\llbracket A \rrbracket_{3, i} \leq \llbracket C \rrbracket_{3, i}$ for all $i$, with strict inequality for at least one $i$, so $A<C$. Also $\llbracket A \rrbracket_{3, i}+\llbracket C \rrbracket_{3, i}=\llbracket 2 B \rrbracket_{3, i}$ for all $i$, so $A+C=2 B$. Finally, let

$$
3^{m}\{a, b, c\}=\{A, B, C\}+\frac{1}{2} .
$$

Then $a+c=2 b$ and $\{a, c\} \subset \mathbb{Q}_{3}^{+}(0,1) \cap \mathbb{Q}_{3}^{+}([1])$.

Claim L. The set $\left(\mathbb{Q}_{3}^{+}(0,1) \backslash \mathbb{Q}_{3}^{+}([1])\right) \cup-\left(\mathbb{Q}_{3}^{+}(0,1) \cap \mathbb{Q}_{3}^{+}([1])\right)$ is midpoint-free.

Proof. By Claims G and J, if the specified set contains a midpoint triple, two members of the triple must have one sign and the third member must have the opposite sign. Suppose $\{a\} \subset \mathbb{Q}_{3}^{+}(0,1) \cap \mathbb{Q}_{3}^{+}([1])$ and $\{b, c\} \subset \mathbb{Q}_{3}^{+}(0,1) \backslash \mathbb{Q}_{3}^{+}([1])$ are such that $-a<b<c$ and $a+2 b=c$. Since $a_{3},(2 b)_{3}$, and $c_{3}$ are eventually periodic, there is an integer $m$ such that their digits in places $i \leq m$ are purely periodic, and

$$
\begin{array}{r}
\llbracket a \rrbracket_{3, i}=1, \quad \llbracket 2 b \rrbracket_{3, i} \in\{0,2\}, \quad \llbracket c \rrbracket_{3, i} \in\{0,1\} \\
\forall i \leq m .
\end{array}
$$

Since $c \notin \mathbb{Q}_{3}^{+}([1])$, there is a $j \leq m$ such that $\llbracket c \rrbracket_{3, j}=0$ and therefore $\llbracket 2 b \rrbracket_{3, i}$ cannot be equal to 0 for all $i \leq m$. On the other hand, $\llbracket 2 b \rrbracket_{3, i}$ cannot be equal to 2 for all $i \leq m$ since $b \notin \mathbb{Q}_{3}^{+}([1])$. Hence the recurring block $[D]$ of $(2 b)_{3}$ contains 0 and 2 , so $\llbracket 2 b \rrbracket_{3, k}=0, \llbracket 2 b \rrbracket_{3, k-1}=2$ for some $k \leq m$. But $\llbracket a \rrbracket_{3, k}=\llbracket a \rrbracket_{3, k-1}=1$, so $\llbracket a \rrbracket_{3, k-1}+\llbracket 2 b \rrbracket_{3, k-1}$ has a "carry over" of 1 , regardless of whether or not the digits in place $k-2$ contribute any "carry over". Then

$$
\llbracket c \rrbracket_{3, k}=\llbracket a \rrbracket_{3, k}+\llbracket 2 b \rrbracket_{3, k}+(1)=2 \notin\{0,1\} .
$$

By this contradiction, there is no midpoint triple of the proposed type. A similar but simpler argument shows that there are no subsets $\{a, b\} \subset \mathbb{Q}_{3}^{+}(0,1) \cap \mathbb{Q}_{3}^{+}([1])$ and $\{c\} \subset$ $\mathbb{Q}_{3}^{+}(0,1) \backslash \mathbb{Q}_{3}^{+}([1])$ such that $-a<-b<c$ and $a=2 b+c$.

Claim $M$. For any positive rational $a \in \mathbb{Q}^{+} \backslash \mathbb{Q}_{3}^{+}([1])$ there is a midpoint triple in $\left(\mathbb{Q}_{3}^{+}(0,1) \backslash \mathbb{Q}_{3}^{+}([1])\right) \cup\{-a\}$, with $-a$ as its lower endpoint. 
Proof. Given $a$, we seek $\{b, c\} \subset \mathbb{Q}_{3}^{+}(0,1) \backslash \mathbb{Q}_{3}^{+}([1])$ such that $-a<b<c$ and $a+2 b=c$. Note that if the triple $\{a, b, c\}$ has all the required properties, the scaled triple $3^{m}\{a, b, c\}$ also has all the required properties, for any integer $m$. Let us assume, without loss of generality, that the digits of $a_{3}$ in negative places are purely periodic, and partitioning into homogeneous blocks $A_{i}$ yields

$$
a_{3}=A_{h} \cdots A_{1} A_{0} \cdot\left[A_{-1} A_{-2} \cdots A_{-k}\right]
$$

for suitable integers $h \geq 0, k>0$. As $a \notin \mathbb{Q}_{3}^{+}([1])$, if $k=1$, then $A_{-1}=0$ so $a \in \mathbb{Z}^{+}$and a suitable set $\{b, c\}$ exists, by Claim D.

Now suppose $k \geq 2$ and $a_{3}$ has at least one negative place digit which is nonzero. If $a_{3}$ has no negative place digit equal to 2 , let $a=A+q$, where $A \in \mathbb{Z}^{+}$and $q \in \mathbb{Q}_{3}^{+}(0,1) \backslash$ $\mathbb{Q}_{3}^{+}([1]), 0<q<1$ are the integer part and fractional part of $a$, respectively. The proof of Claim $\mathrm{D}$ yields integers $B, C \in \mathbb{Z}_{3}^{+}(0,1)$, such that $\{-A, B, C\}$ is a midpoint triple. Choose $b=B, c=C+q$. Then

$$
\{-a, b, c\}=\{-A-q, B, C+q\}
$$

is a midpoint triple with $\{b, c\} \subset \mathbb{Q}_{3}^{+}(0,1) \backslash \mathbb{Q}_{3}^{+}([1])$.

Finally, suppose $A_{-j}=2^{\times n}$ for at least one $j>0$. Define homogeneous blocks $B_{i}$ and $C_{i}$ for all $i$ as follows:

$$
\begin{aligned}
& A_{i}=0^{\times n} \Longrightarrow B_{i}=1^{\times n}, \quad C_{i}=0^{\times n} \\
& A_{i}=1^{\times n} \Longrightarrow B_{i}=1^{\times n}, \quad C_{i}=1^{\times n} \\
& A_{i}=2^{\times n} \Longrightarrow B_{i}=0^{\times n}, \quad C_{i}=0^{\times n} .
\end{aligned}
$$

Assuming a carry over digit equal to 1 , note that checks as in the proof of Claim D justify the calculations

$$
0^{\times n}+2^{\times n}+(1)=(1) 0^{\times n}, \quad 1^{\times n}+2^{\times n}+(1)=(1) 1^{\times n},
$$

so in all cases we have $A_{i}+2 B_{i}+(1)=(1) C_{i}$. Choose

$$
\begin{aligned}
& b_{3}=B_{h} \cdots B_{1} B_{0} \cdot\left[B_{-1} B_{-2} \cdots B_{-k}\right], \\
& c_{3}=(1) C_{h} \cdots C_{1} C_{0} \cdot\left[C_{-1} C_{-2} \cdots C_{-k}\right] .
\end{aligned}
$$

Then $a+2 b=c$ and $\{b, c\} \subset \mathbb{Q}_{3}^{+}(0,1)$. Also $B_{-j}=C_{-j}=0^{\times n}$ ensures that $\{b, c\} \subset \mathbb{Q}_{3}^{+}(0,1) \backslash \mathbb{Q}_{3}^{+}([1])$, as required.

For instance, beginning with

$$
a=2 \cdot[201]_{3}=\frac{71}{26} \in \mathbb{Q}^{+} \backslash \mathbb{Q}_{3}^{+}([1]),
$$

the proof of Claim M constructs a midpoint triple $\{-a, b, c\}$ with

$$
\begin{aligned}
& b=0 \cdot[011]_{3}=\frac{2}{13} \in \mathbb{Q}_{3}^{+}(0,1) \backslash \mathbb{Q}_{3}^{+}([1]), \\
& c=10 \cdot[001]_{3}=\frac{79}{26} \in \mathbb{Q}_{3}^{+}(0,1) \backslash \mathbb{Q}_{3}^{+}([1]) .
\end{aligned}
$$

However, if we begin with

$$
a=2 \cdot[101]_{3}=\frac{31}{13} \in \mathbb{Q}^{+} \backslash \mathbb{Q}_{3}^{+}([1]),
$$

the construction falls back to Claim $\mathrm{D}$, resulting in $b=1$ and $c=57 / 13$.

It now follows from Claims I, K, L, and $\mathrm{M}$ that the set

$$
\left(\mathbb{Q}_{3}^{+}(0,1) \backslash \mathbb{Q}_{3}^{+}([1])\right) \cup-\left(\mathbb{Q}_{3}^{+}(0,1) \cap \mathbb{Q}_{3}^{+}([1])\right)
$$

is a maximal midpoint-free subset of $\mathbb{Q}$. This settles midpoint questions about $\mathbb{Q}_{3}^{+}(0,1)$ as a subset of $\mathbb{Q}$.

What is the situation for $\mathbb{Q}_{3}^{+}(0,2)$ ? If $q \in \mathbb{Q}_{3}^{+}(0,2)$ then the recurring block of $q_{3}$ must have at least one digit equal to 0 . Hence, there must be at least one digit equal to 0 in the recurring block of $(q / 2)_{3}$, so $q / 2 \in \mathbb{Q}_{3}^{+}(0,1) \backslash \mathbb{Q}_{3}^{+}([1])$ : it follows that $2\left(\mathbb{Q}_{3}^{+}(0,1) \backslash \mathbb{Q}_{3}^{+}([1])\right)=\mathbb{Q}_{3}^{+}(0,2)$. Again, if there is some $m \in \mathbb{Z}^{+}$, such that $3^{m} q \in \mathbb{Z}_{3}^{+}(0,2)+1$, it follows that $q_{3}$ is a terminating representation with trailing digit 1 and all other digits in $\{0,2\}$. Hence,

$$
\frac{q}{2} \in \mathbb{Q}_{3}^{+}(0,1) \cap \mathbb{Q}_{3}^{+}([1]) .
$$

The converse also holds, so

$$
2\left(\mathbb{Q}_{3}^{+}(0,1) \cap \mathbb{Q}_{3}^{+}([1])\right)=\mathbb{Q}_{3}^{+}(0,2 ; 1),
$$

where $\mathbb{Q}_{3}^{+}(0,2 ; 1)$ is the set of positive rationals which have a terminating base 3 representation with trailing digit 1 and all other digits in $\{0,2\}$, so

$$
\mathbb{Q}_{3}^{+}(0,2 ; 1)=\bigcup_{i \in \mathbb{Z}^{+}} 3^{-i}\left(\mathbb{Z}_{3}^{+}(0,2)+1\right) .
$$

In passing, note that $\mathbb{Z}_{3}^{+}(0,2)+1=\mathbb{Q}_{3}^{+}(0,2 ; 1) \cap \mathbb{Z}^{+}$. By doubling, it now follows from Claim $L$ that the set

$$
\mathbb{Q}_{3}^{+}(0,2) \cup-\mathbb{Q}_{3}^{+}(0,2 ; 1)
$$

is midpoint-free.

Claim $N$. Any rational $b \in \mathbb{Q}^{+} \backslash \mathbb{Q}_{3}^{+}(0,2)$ is the midpoint of a triple with both its endpoints in $\mathbb{Q}_{3}^{+}(0,2)$.

Proof. If $b \in \mathbb{Q}^{+} \backslash \mathbb{Q}_{3}^{+}(0,2)$, then $b / 2 \notin \mathbb{Q}_{3}^{+}(0,1)$, so $b / 2$ is the midpoint of a triple with endpoints in $\mathbb{Q}_{3}^{+}(0,1) \backslash \mathbb{Q}_{3}^{+}([1])$, by Claim I. By doubling, $b$ is the midpoint of a triple with both its endpoints in the set $\mathbb{Q}_{3}^{+}(0,2)$.

Claim 0 . For any rational $a \in \mathbb{Q}^{+} \backslash \mathbb{Q}_{3}^{+}(0,2 ; 1)$, there is a midpoint triple in $\mathbb{Q}_{3}^{+}(0,2) \cup\{-a\}$ with $-a$ as its lower endpoint.

Proof. If $a \in \mathbb{Q}^{+} \backslash \mathbb{Q}_{3}^{+}(0,2 ; 1)$ and $a>0$, then $a / 2 \notin \mathbb{Q}_{3}^{+}([1])$, so there is a midpoint triple in $\mathbb{Q}_{3}^{+}(0,1) \backslash \mathbb{Q}_{3}^{+}([1]) \cup\{-a / 2\}$ with $-a / 2$ as its lower endpoint, by Claim M. By doubling, $\mathbb{Q}_{3}^{+}(0,2) \cup\{-a\}$ has a midpoint triple with lower endpoint $-a$.

The preceding results now fully establish the following.

Theorem 4. The two sets

$$
\begin{gathered}
\left(\mathbb{Q}_{3}^{+}(0,1) \backslash \mathbb{Q}_{3}^{+}([1])\right) \cup-\left(\mathbb{Q}_{3}^{+}(0,1) \cap \mathbb{Q}_{3}^{+}([1])\right), \\
\mathbb{Q}_{3}^{+}(0,2) \cup-\mathbb{Q}_{3}^{+}(0,2 ; 1)
\end{gathered}
$$

are both maximal midpoint-free subsets of $\mathbb{Q}$. 


\section{Midpoint-Free Subsets of $\mathbb{R}^{+}$and $\mathbb{R}$}

Now consider $\mathbb{R}_{3}^{+}(0,1)$ and $\mathbb{R}_{3}^{+}(0,2)$. These sets are uncountable, while their maximal rational subsets $\mathbb{Q}_{3}^{+}(0,1)$ and $\mathbb{Q}_{3}^{+}(0,2)$ are countable, yet no essentially new considerations arise in extending the results of the previous two sections from $\mathbb{Q}^{+}$to $\mathbb{R}^{+}$and from $\mathbb{Q}$ to $\mathbb{R}$. Noting that $\mathbb{R}_{3}^{+}([1])=$ $\mathbb{Q}_{3}^{+}([1])$ and $\mathbb{R}_{3}^{+}(0,2 ; 1)=\mathbb{Q}_{3}^{+}(0,2 ; 1)$, it suffices to state the end results for this wider context.

Theorem 5 . The sets $\mathbb{R}_{3}^{+}(0,1) \backslash \mathbb{R}_{3}^{+}([1])$ and $\mathbb{R}_{3}^{+}(0,2)$ are both maximal midpoint-free subsets of $\mathbb{R}^{+}$.

Theorem 6. The two sets

$$
\begin{gathered}
\left(\mathbb{R}_{3}^{+}(0,1) \backslash \mathbb{R}_{3}^{+}([1])\right) \cup-\left(\mathbb{R}_{3}^{+}(0,1) \cap \mathbb{R}_{3}^{+}([1])\right) \\
\mathbb{R}_{3}^{+}(0,2) \cup-\mathbb{R}_{3}^{+}(0,2 ; 1)
\end{gathered}
$$

are both maximal midpoint-free subsets of $\mathbb{R}$.

\section{Conflict of Interests}

The author declares that there is no conflict of interests regarding the publication of this paper.

\section{References}

[1] N. Sloane, OEIS-Online Encyclopedia of Integer Sequences, Note A003002 and A005836 in particular; many relevant links are included.

[2] R. K. Guy, Unsolved Problems in Number Theory, Springer, New York, NY, USA, 3rd edition, 2004.

[3] J. Dybizbański, "Sequences containing no 3-term arithmetic progressions," Electronic Journal of Combinatorics, vol. 19, no. 2, pp. 1-15, 2012.

[4] D. Wells, The Penguin Dictionary of Curious and Interesting Numbers, Penguin, London, UK, 1986.

[5] S. Yates, "Prime divisors of repunits," Journal of Recreational Mathematics, vol. 8, no. 1, pp. 33-38, 1975.

[6] J. Brillhart, D. H. Lehmer, J. L. Selfridge, B. Tuckerman, and S. Wagstaff Jr., "Factorizations of $b^{n} \pm 1, b=2,3,5,6,7,10,11,12$ up to high powers," Contemporary Mathematics, vol. 22, 1983.

[7] P. Erdős and P. Turán, "On some sequences of integers," Journal of the London Mathematical Society, vol. 28, pp. 104-109, 1936. 


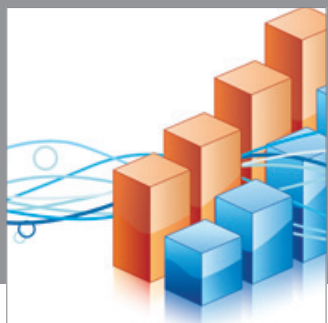

Advances in

Operations Research

mansans

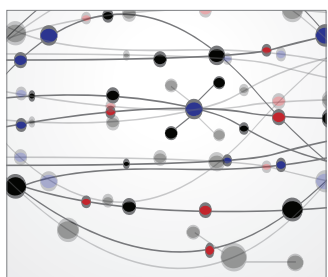

The Scientific World Journal
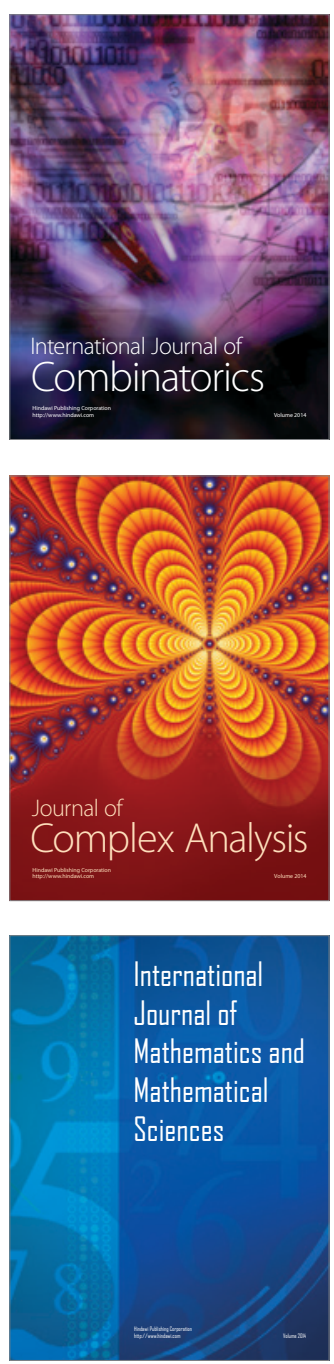
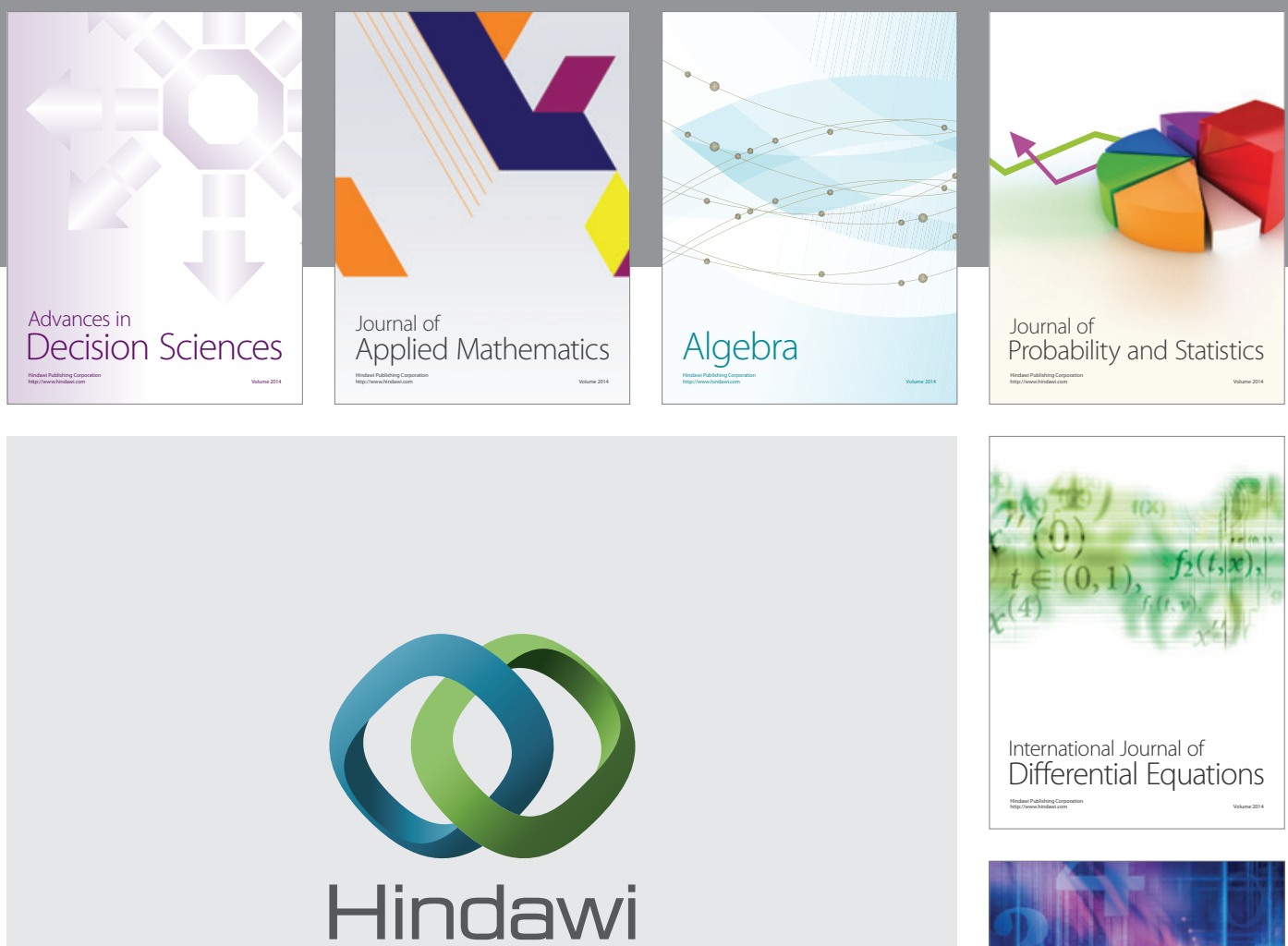

Submit your manuscripts at http://www.hindawi.com
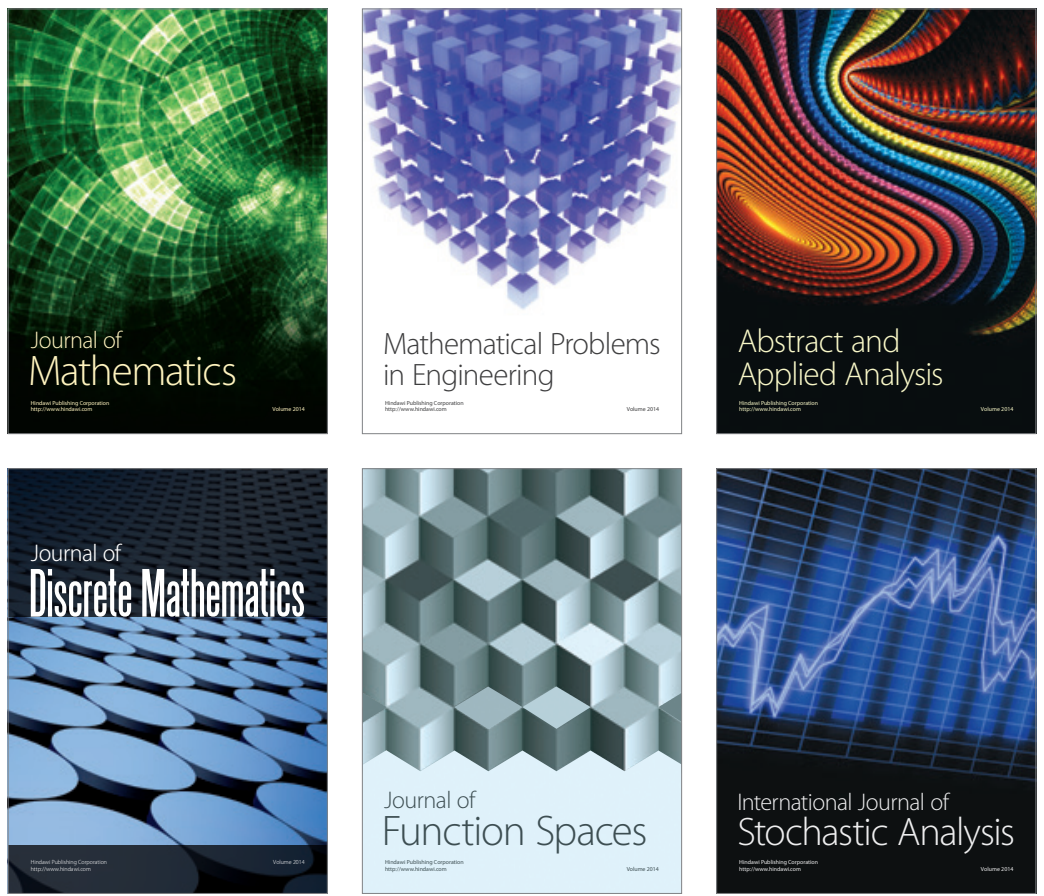

Journal of

Function Spaces

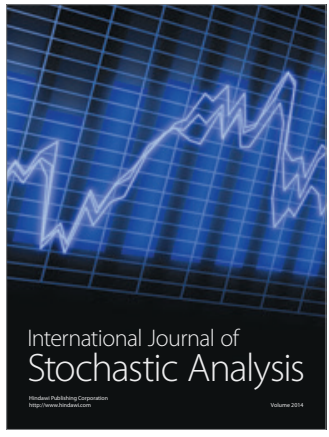

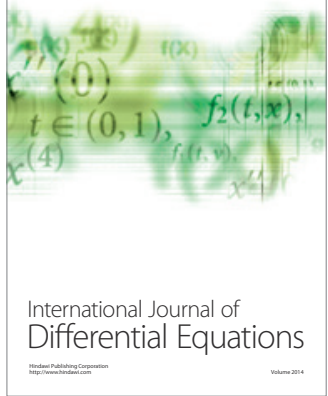
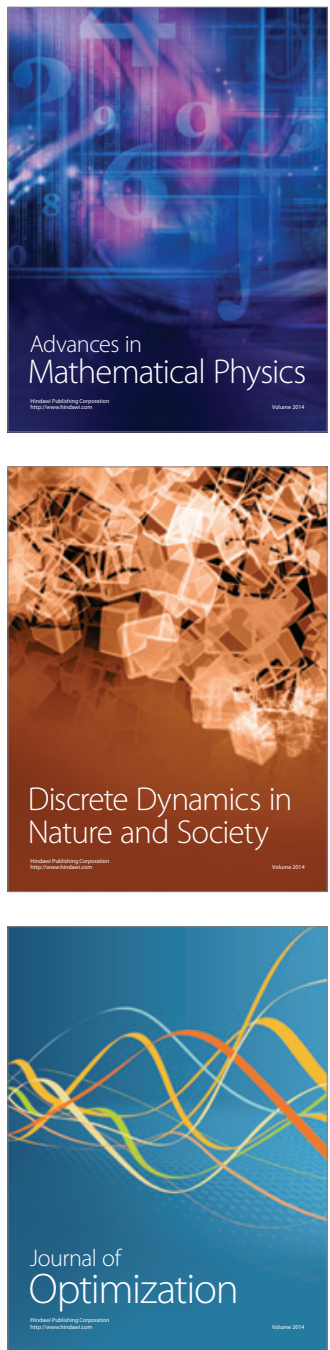